\title{
Gender differences in health-related quality of life of Australian chronically-ill adults: patient and physician characteristics do matter
}

\author{
Upali W Jayasinghe ${ }^{1,2^{*}}$, Mark F Harris ${ }^{1}$, Jane Taggart ${ }^{1}$, Bettina Christl ${ }^{1}$ and Deborah A Black ${ }^{3}$
}

\begin{abstract}
Background: The aims of this study were to explore the health-related quality of life (HRQoL) in a large sample of Australian chronically-ill patients (type 2 diabetes and/or hypertension/ischaemic heart disease), to investigate the impact of characteristics of patients and their general practitioners on their HRQoL and to examine clinically significant differences in HRQOL among males and females.
\end{abstract}

Methods: This was a cross-sectional study with 193 general practitioners and 2181 of their chronically-ill patients aged 18 years or more using the standard Short Form Health Survey (SF-12) version 2. SF-12 physical component score (PCS-12) and mental component score (MCS-12) were derived using the standard US algorithm. Multilevel regression analysis (patients at level 1 and general practitioners at level 2) was applied to relate PCS-12 and MCS-12 to patient and general practitioner (GP) characteristics.

Results: Employment was likely to have a clinically significant larger positive effect on HRQoL of males (regression coefficient (B) $(P C S-12)=7.29, P<0.001$, effect size $=1.23$ and $B($ MCS-12 $)=3.40, P<0.01$, effect size $=0.55)$ than that of females $(B(P C S-12)=4.05, P<0.001$, effect size $=0.78$ and $B($ MCS-12) $=1.16, P>0.05$, effect size $=0.16)$. There was a clinically significant difference in $\mathrm{HRQOL}$ among age groups. Younger men $(<39$ years) were likely to have better physical health than older men ( $>59$ years, $B=-5.82, P<0.05$, effect size $=0.66$ ); older women tended to have better mental health $(B=5.62, P<0.001$, effect size $=0.77)$ than younger women. Chronically-ill women smokers reported clinically significant $(B=-3.99, P<0.001$, effect size $=0.66)$ poorer mental health than women who were non-smokers. Female GPs were more likely to examine female patients than male patients (33\% vs. $15 \%, P<0.001)$ and female patients attending female GPs reported better physical health $(B=1.59, P<0.05$, effect size $=0.30$ ).

Conclusions: Some of the associations between patient characteristics and SF-12 physical and/or mental component scores were different for men and women. This finding underlines the importance of considering these factors in the management of chronically-ill patients in general practice. The results suggest that chronically ill women attempting to quit smoking may need more psychological support. More quantitative studies are needed to determine the association between GP gender and patient gender in relation to HRQoL.

Keywords: Quality of life, Patient and physician characteristics, SF-12 version 2, Physical component score, Mental component score, Multilevel regression analysis

\footnotetext{
* Correspondence: upali.jay@unsw.edu.au

${ }^{1}$ Centre for Primary Health Care and Equity, University of New South Wales, Sydney, New South Wales, Australia

${ }^{2}$ Westmead Breast Cancer Institute, Westmead, New South Wales, Australia

Full list of author information is available at the end of the article
} 


\section{Background}

In 2004, 77\% of Australians reported having at least one long-term medical condition [1]. The management of chronic illness has become a major focus in general practice in Australia, both because of its prevalence and because of the opportunity for general practice to intervene early to improve quality of life, prevent disability, and reduce hospital use [2].

The SF-36 and SF-12 are widely used measures of health-related quality of life (HRQoL). Investigators from numerous countries representing diverse cultures have determined that both measures are sensitive to differences in a number of socio-demographic and clinical variables, including gender [3,4], age [2,3], income [4-6], employment $[2,4,5]$, education [5,6], marital status [2,7], satisfaction with care [2], smoking [8-10], and number of chronic conditions [6]. Several studies have found women to have lower HRQoL scores than men $[11,12]$ and a few studies have found that smoking had a greater negative effect on HRQoL of women than men [13]. Employment had a stronger association with the quality of life of males than that of females [2]. In addition to patient factors, we also included the socio-demographic characteristics of the general practitioners (GPs). Earlier research has shown that female GPs provided longer consultations and saw patients with different health problems than their male colleagues $[14,15]$. Gender is one of the many factors that impact upon the doctorpatient interaction [16]. From the above findings, patient and GP characteristics tended to interact with gender of patients in predicting HRQoL, so we decided to analyse data separately for males and females.

In this study, we investigated the relationship between patient or GP characteristics and HRQoL in a large sample of chronically-ill Australian adults from two states and the Australian Capital Territory, using SF-12 version 2. No one we know of has investigated the impact of characteristics of general practitioners on their patients' HRQoL before. Based on the findings and identified gaps in the literature, the following research questions were posed:

1) What are the differences in patient gender among subgroups of patient age, socio-economic status (home and car ownership, education and employment), marital status, smoking status, number of chronic diseases, satisfaction with care, GP gender, country of graduation, age (years as a GP), years in the current practice, GP's practice size and location?

2) Are these factors independent predictors of HRQoL for male and female patients?

3) Are there any clinically significant differences in HRQoL among patient and GP subgroups?

\section{Methods}

\section{Participants}

The Teamwork study was a cluster randomised trial involving 60 practices in urban and rural New South Wales (NSW), Victoria and the Australian Capital Territory between July 2006 and June 2008 [17]. This study aimed to evaluate the impact of facilitating an enhanced role for non-GP staff in the management of patients with chronic illness. Chronic illnesses are diseases of long duration and generally slow progression. In this study we included chronic ill patients suffering from three conditions (type 2 diabetes and/or hypertension/ ischaemic heart disease) for more than three months duration as diagnosed by the GP. In the Teamwork study, 3349 type 2 diabetes and/or hypertension/ischaemic heart disease patients aged 18 years or more attending 60 practices were invited by their GPs to participate, and of these, 2642 (79\%) consented to participate. Patients were eligible to participate in the study if they had attended the practice in the preceding 12 months. For 2181 (1018 males and 1163 females) of the participating patients, their GPs $(n=193)$ had completed a clinical care interview (CCI) which provided information on the GP's socio-demographic and practice characteristics. In this paper, a cross-sectional analysis of baseline data was conducted. Pearson Chi-Squared tests indicated that the proportions of gender, age, employment, education, home and car ownership, marital status, smoking status, and number of conditions were similar between all patients who consented to participate $(n=2642$, $97 \%$ of whom provided patient characteristics) and the subgroup of patients whose GPs had completed the CCI ( $n=2181)$.

A priori sample size calculation on the SF-12 mental component score predicted that, after adjustment for clustering, an average sample size of 30 patients from each of the 60 practices would have sufficient power $(1-\beta=0.8$ and $\alpha=0.05)$ to detect an effect size of 0.13 between smokers and non-smokers, assuming that about $10 \%$ of the patients were smokers (previous studies on SF-12 indicated a cluster effect (ICC = intra-cluster correlation) of 0.011 for the MCS-12 score [2]). Similarly 10 female patients attending each of 90 male or female GPs would have sufficient power $(1-\beta=0.8$ and $\alpha=0.05)$ to detect an effect size of 0.15 for physical component scores between male and female GPs.

\section{Ethics}

Ethics approval for the study was obtained from the University of New South Wales (UNSW) Human Research Ethics Committee, and we obtained full informed written consent from participants. 


\section{Instruments}

The standard SF-12 version 2 is a 12 -item questionnaire measuring physical and mental health $[6,18]$. Use of the SF-12 version 2 has been recommended in preference to the original version 1 form for all new studies [19]. It is an abbreviated form of the SF-36 Health Survey, which is one of the most widely used instruments for assessing HRQoL [6]. Both instruments produce eight dimensions of health: physical functioning (PF), role limitations due to physical problems (RF), bodily pain (BP), general health $(\mathrm{GH})$, vitality (VT), social functioning (SF), role limitation due to emotional problems (RE), and mental health $(\mathrm{MH})$ [20,21]. They also produce two summary scores - the Physical Component Summary (PCS) and the Mental Health Component Summary (MCS) - and have been validated for use in the USA, UK and many other European countries for large-scale health measurement and monitoring [6,21]. For ease of interpretation, scores are standardized to population norms, with the mean score set at $50(\mathrm{SD}=10)$; higher scores indicate better health. The SF-12 has been shown to have good validity and reliability [19]. Previous research supports the use of the standard SF-12 in Australian settings, and it has been validated for Australia using standard USderived scoring of the SF-12 summary scores [2,22,23].

Patients were invited by their GPs to participate and were mailed the SF12 and the Patient Assessment of Chronic Illness Care (PACIC) questionnaire with some additional demographic questions including smoking status and chronic medical condition/conditions. A reminder survey was mailed if there was no response after 4 weeks. The PACIC is an instrument that measures patients' assessment of the care they receive. The psychometric properties of the PACIC have been evaluated in Australia [17].

\section{Data and variables}

The dependent variables were PCS-12 and MCS-12 scores. The GP characteristics included were gender, country of graduation, number of years in the current practice, number of years as a GP, and practice size and location (urban/rural). Both number of years in the current practice and number of years as a GP were divided into tertiles (low, middle, high values). The socio-demographic characteristics of patients studied were gender, age, home and car ownership, education, employment, marital status, smoking status, number of chronic diseases, and overall satisfaction with care (PACIC) (Table 1). Patients were asked if they were current smokers ("Do you currently smoke?"). Home and car ownership can be considered markers of economic status [24].

\section{Statistical analyses}

Summary physical (PCS-12) and mental (MCS-12) components were constructed using the standard SF-12 version 2 US algorithm which is empirically derived from the data of a US general population survey [19]. The dimensions as documented by Kontodimopoulos et al. [25] and Ware et al. [19] were confirmed and validated for Australia using standard US-derived scoring of the SF-12 summary scores [2].

Univariate analyses were carried out using IBM SPSS version 20 (Tables 1 and 2). First, we examined the association between the independent variables and patient gender using the Pearson chi-squared test. Mean unadjusted scores of PCS-12 and MCS-12 among male and female patients in each category of the independent variables were compared using t-tests.

We computed Cohen's d effect sizes. Cohen defined an effect size of 0.20 as small, 0.50 as moderate, and 0.80 or greater as large [26]. An effect size of more than 0.5 or half a standard deviation is considered to be clinically significant $[2,3]$.

\section{Multilevel models}

Multilevel regression models were used for male and female patients with two dimensions (physical and mental component scores) as continuous dependent variables and general practice and patient characteristics as independent variables. Multilevel analysis used MLwiN 2.25 [27] adjusted for clustering of patients (level 1) within GPs (level 2) [2,4]. Initially, we fitted a baseline variance component model (no independent variables) for each of the response variables followed by the main model. The main model expands the baseline model by including patient and GP characteristics. Parameter estimates of fixed effects were tested for significance using a $t$-test, determined by dividing the estimated coefficients by their standard errors. The significance of the random variance estimates was assessed using the Wald joint chi-squared test [27].

\section{Multiple imputations}

We used multiple imputations to address the potential bias and loss of precision that could result from complete-case analysis. Multiple datasets were created, as each missing value was replaced with a set of random plausible values conditional on known covariates and known distributional information. REALCOM-IMPUTE software with MLwiN was used for the analysis [28]. A total run length of 6500 iterations was used with imputations made after every $500^{\text {th }}$ iteration following 1500 burn-in iterations to ensure that the imputations were independent. Ten completed datasets were imputed and the model of interest was fitted to each of these datasets. The results from the ten imputed datasets were combined using Rubin's rule [29]. Analyses were conducted with $\{($ males $(\mathrm{n}=1018)$, females $(\mathrm{n}=1163)\}$ and without $\{($ males $(n=768)$, females $(n=823)$, after listwise 
Table 1 Distribution of patient gender according to patient and general practitioner characteristics for 2181 chronically-ill adults

\begin{tabular}{lccc}
\hline Variable (definition) & \multicolumn{2}{c}{ Gender } & \\
\cline { 2 - 3 } & $\begin{array}{c}\text { Male } \\
(\mathrm{n}=1018)\end{array}$ & $\begin{array}{c}\text { Female } \\
(\mathrm{n}=1163)\end{array}$ & p-value ${ }^{1}$ \\
\cline { 2 - 3 } & Number $\%$ & Number $\%$ & \\
\hline
\end{tabular}

Characteristics of patients

Age, years

18-39

40-59

$>59$

$\begin{array}{cccc}18 & 1.8 & 50 & 4.3 \\ 274 & 27.0 & 322 & 27.8 \\ 722 & 71.2 & 788 & 67.9\end{array}$

Health status

Good

Home ownership

Own home and car

Own home only

Own car only

Rent and do not own a car

Education

Degree/Diploma
Elementary/High school

Employment

Employed

Retired

Unemployed $^{2}$

Marital status

Married

(married/cohabiting)

Unmarried $^{3}$

Disease

One condition
Two or more conditions
Smoker
Yes
No

Overall PACIC score

High (above median)

Low (below median)

Characteristics of general practitioner $(G P)(n=193)$

Gender of GP

$$
\text { Male }(n=121)
$$

Female $(n=72)$

Country of graduation

$$
\text { Australia }(n=143)
$$

$\begin{array}{lllll}603 & 60.4 & 631 & 55.4 & 0.019 \\ 396 & 39.6 & 509 & 44.6 & \end{array}$

39.6

$\begin{array}{llll}795 & 79.2 & 797 & 69.3\end{array}$

$\begin{array}{llll}136 & 13.5 & 154 & 13.4\end{array}$

$\begin{array}{llll}51 & 5.1 & 78 & 6.8\end{array}$

$\begin{array}{llll}370 & 36.6 & 356 & 30.9\end{array}$

$\begin{array}{llll}642 & 63.4 & 795 & 69.1\end{array}$

0.006

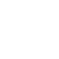

51

14

$<0.001$ $\begin{array}{llll}22 & 2.2 & 121 & 10.5\end{array}$
Table 1 Distribution of patient gender according to patient and general practitioner characteristics for 2181 chronically-ill adults (Continued)

\begin{tabular}{llllll} 
Overseas $(n=49)$ & 184 & 18.1 & 248 & 21.4 & \\
Years in the current practice & & & & & \\
$<6(n=85)$ & 337 & 33.3 & 462 & 40.1 & 0.001 \\
$6-15(n=56)$ & 266 & 26.3 & 307 & 26.7 & \\
$>15(n=47)$ & 408 & 40.4 & 382 & 33.2 & \\
Years as a GP & & & & & \\
$<19(n=77)$ & 288 & 28.5 & 427 & 37.3 & $<0.001$ \\
$19-24(n=48)$ & 319 & 31.6 & 366 & 32.0 & \\
$>24(n=54)$ & 403 & 39.9 & 352 & 30.7 & \\
$\begin{array}{l}\text { Works in a small practice } \\
1-3 \text { GPs) }(n=39)\end{array}$ & 435 & 42.7 & 487 & 41.9 & 0.686 \\
Works in a large practice & 583 & 57.3 & 676 & 58.1 & \\
$>3$ GPs) $(n=154)$ & & & & & \\
ocation of work & & & & & \\
$\quad$ Urban ( $n=101)$ & 503 & 49.4 & 524 & 45.1 & 0.042 \\
Rural $(n=92)$ & 515 & 50.6 & 639 & 54.9 & \\
\hline
\end{tabular}

Notes: ${ }^{1} p$-values are for comparison of categories of each variable by gender using Pearson's chi-square test.

${ }^{2}$ Includes patients looking for work, studying full-time, looking after family and unable to work due to sickness or disability.

${ }^{3}$ Includes single/separated/divorced/widowed.

Unknowns: Age $=7$, Home and car ownership $=27$, Education $=18$,

Employment $=18$, Marital status $=69$, Disease $=50$, Smoking $=46$, Overall

PACIC score $=94$, Years as a GP $=26$, Years in the practice $=19$.

Patient characteristics were collected independently using PACIC for the same respondents.

$\mathrm{n}=$ number of GPs.

deletion of missing component scores and covariates\} the cases with imputed results (Tables $3 \& 4$ ). There were no meaningful differences in the results and therefore those for the entire sample are discussed in this paper.

\section{Results}

The mean ages of male and female patients were 64 years (range 24-90) and 63 years (range 18-93) respectively.

The gender comparison for socio-demographic characteristics of patients and GPs is presented in Table 1. Female patients tended to have lower socio-economic status (13\% more unemployed, $10 \%$ less owner-occupiers with cars, $6 \%$ more lower-educated) than male patients (Table 1; $\mathrm{P}<0.001)$. Married patients were more likely to be males $(78 \%$ vs $64 \%, \mathrm{P}<0.001)$. Male patients were more likely than females to have more than one chronic disease $(\mathrm{P}<0.001)$. Female GPs were more likely to have female than male patients $(33 \%$ vs. $15 \%, \mathrm{P}<0.001)$ and the proportions were $37 \%$ vs. $18 \%(\mathrm{P}<0.001)$ without solo practices. Female patients tended to be under the care of younger (practicing for $<19$ years) GPs than male patients $(37 \%$ vs $29 \%, \mathrm{P}<0.001)$. 
Table 2 Unadjusted mean and standard deviation of PCS-12 and MCS-12 scores by characteristics of practices and patients

\begin{tabular}{|c|c|c|c|c|c|c|}
\hline \multirow{3}{*}{ Variable (definition) } & \multicolumn{3}{|c|}{ PCS-12 } & \multicolumn{3}{|c|}{ MCS-12 } \\
\hline & Male & Female & & Male & Female & \\
\hline & Mean (SD) & Mean (SD) & p-value ${ }^{1}$ & Mean (SD) & Mean (SD) & p-value ${ }^{1}$ \\
\hline Overall score & $43.0(11.7)$ & $42.3(12.4)$ & 0.206 & $50.0(10.9)$ & $48.3(11.2)$ & 0.002 \\
\hline \multicolumn{7}{|l|}{ Characteristics of patients } \\
\hline \multicolumn{7}{|l|}{ Age, years } \\
\hline $18-39$ & $49.3(7.7)$ & $46.3(11.3)$ & 0.246 & $46.7(10.7)$ & $41.9(11.9)$ & 0.154 \\
\hline $40-59$ & $46.1(10.7)$ & $45.9(11.9)$ & 0.870 & $48.2(11.0)$ & $45.3(11.8)$ & 0.005 \\
\hline$>59$ & $41.6(11.8)$ & $40.5(12.4)$ & 0.106 & $50.8(10.7)$ & $50.0(10.4)$ & 0.214 \\
\hline \multicolumn{7}{|l|}{ Health status } \\
\hline Good & $48.1(9.1)$ & 48.3(9.4) & 0.822 & $53.0(8.7)$ & $52.0(9.1)$ & 0.059 \\
\hline Poor & $35.2(10.8)$ & $34.5(11.6)$ & 0.397 & $45.4(12.2)$ & $43.4(11.7)$ & 0.025 \\
\hline \multicolumn{7}{|l|}{ Home ownership } \\
\hline Own home and car & 43.8(11.4) & $44.1(12.1)$ & 0.605 & $50.9(10.4)$ & $49.3(10.7)$ & 0.007 \\
\hline Own home only & $38.2(11.4)$ & $37.9(12.1)$ & 0.939 & 47.9(10.8) & $47.2(11.4)$ & 0.820 \\
\hline Own car only & $42.4(12.1)$ & $39.7(12.3)$ & 0.081 & 47.1(11.6) & $45.7(11.0)$ & 0.323 \\
\hline Rent and do not own a car & $34.7(11.5)$ & $34.5(12.5)$ & 0.935 & $42.3(11.8)$ & $44.5(13.9)$ & 0.423 \\
\hline \multicolumn{7}{|l|}{ Education } \\
\hline Degree/Diploma & $45.7(10.3)$ & $44.8(11.9)$ & 0.284 & $51.7(10.3)$ & 48.0(10.6) & $<0.001$ \\
\hline Elementary/High school & $41.5(12.2)$ & $41.2(12.5)$ & 0.688 & $49.2(11.1)$ & $48.3(11.4)$ & 0.199 \\
\hline \multicolumn{7}{|l|}{ Employment } \\
\hline Employed & $48.0(9.2)$ & $48.2(10.7)$ & 0.785 & $49.7(9.9)$ & $47.1(10.9)$ & 0.003 \\
\hline Retired & $41.5(11.6)$ & $40.5(11.8)$ & 0.224 & $52.0(10.1)$ & $51.0(9.9)$ & 0.152 \\
\hline Unemployed ${ }^{2}$ & $35.6(11.9)$ & $38.9(13.2)$ & 0.020 & $43.7(13.3)$ & $45.3(12.3)$ & 0.248 \\
\hline \multicolumn{7}{|l|}{ Marital status } \\
\hline Married (married/cohabiting) & $43.4(11.5)$ & $43.4(12.2)$ & 0.998 & $50.6(10.5)$ & 49.4(10.8) & 0.061 \\
\hline Unmarried $^{3}$ & $41.4(12.4)$ & $39.9(12.6)$ & 0.163 & $47.6(12.1)$ & $46.2(11.6)$ & 0.225 \\
\hline \multicolumn{7}{|l|}{ Disease } \\
\hline One condition & $45.1(11.6)$ & $43.6(12.4)$ & 0.035 & $50.3(10.5)$ & $49.0(11.0)$ & 0.052 \\
\hline Two or more conditions & $40.3(11.1)$ & $39.5(12.2)$ & 0.378 & 49.5(11.3) & $47.2(11.1)$ & 0.010 \\
\hline \multicolumn{7}{|l|}{ Smoker } \\
\hline Yes & $41.3(11.8)$ & $41.0((12.3)$ & 0.850 & $48.0(11.5)$ & $41.9(12.1)$ & 0.001 \\
\hline No & 43.3(11.6) & $42.4(12.5)$ & 0.157 & $50.1(10.8)$ & 49.1(10.8) & 0.056 \\
\hline \multicolumn{7}{|l|}{ Overall PACIC score } \\
\hline High (above median) & $42.6(11.7)$ & $41.1(12.6)$ & 0.070 & $50.3(10.7)$ & 48.4(10.6) & 0.009 \\
\hline Low (below median) & $43.3(11.7)$ & $43.1(12.3)$ & 0.813 & $49.3(11.1)$ & 47.6(11.6) & 0.030 \\
\hline \multicolumn{7}{|c|}{ Characteristics of general practitioner (GP) } \\
\hline \multicolumn{7}{|l|}{ Gender of GP } \\
\hline Male & $42.8(11.8)$ & $41.1(12.5)$ & 0.014 & 49.9(10.8) & $48.5(11.3)$ & 0.017 \\
\hline Female & 44.4(10.7) & $44.8(11.9)$ & 0.716 & $50.1(11.3)$ & $47.8(11.0)$ & 0.050 \\
\hline \multicolumn{7}{|l|}{ Country of graduation } \\
\hline Australia & $42.9(11.8)$ & $42.3(12.5)$ & 0.366 & $50.4(10.9)$ & $48.4(11.1)$ & 0.001 \\
\hline Overseas & $43.8(11.0)$ & $42.4(12.2)$ & 0.292 & $48.2(10.8)$ & $47.8(11.5)$ & 0.718 \\
\hline
\end{tabular}




\begin{tabular}{|c|c|c|c|c|c|c|}
\hline \multicolumn{7}{|l|}{ Years in the current practice } \\
\hline$<6$ & $42.8(11.1)$ & $43.0(12.2)$ & 0.799 & 49.3(10.8) & $47.3(11.3)$ & 0.028 \\
\hline $6-15$ & $42.6(11.8)$ & $41.7(12.9)$ & 0.449 & $49.4(12.4)$ & $49.6(11.1)$ & 0.853 \\
\hline$>15$ & $43.6(12.1)$ & $42.0(12.1)$ & 0.094 & $51.0(9.9)$ & $48.5(11.0)$ & 0.003 \\
\hline \multicolumn{7}{|l|}{ Years as a GP } \\
\hline$<19$ & $44.0(11.0)$ & $43.1(12.2)$ & 0.403 & $49.2(10.7)$ & $48.1(11.3)$ & 0.242 \\
\hline $19-24$ & $42.3(12.1)$ & $42.6(12.2)$ & 0.819 & $50.3(11.0)$ & $48.9(11.0)$ & 0.118 \\
\hline$>24$ & $43.1(11.7)$ & $41.2(12.9)$ & 0.068 & $50.2(10.8)$ & 47.9(11.6) & 0.013 \\
\hline Works in a small practice (1-3 GPs) & $42.8(12.4)$ & $41.9(13.0)$ & 0.312 & $49.0(11.3)$ & $47.5(11.6)$ & 0.082 \\
\hline Works in a large practice (> 3 GPs) & $43.2(11.1)$ & $42.6(12.0)$ & 0.437 & $50.7(10.5)$ & 48.9(10.8) & 0.006 \\
\hline \multicolumn{7}{|l|}{ Location of work } \\
\hline Urban & $43.7(11.4)$ & $42.2(12.0)$ & 0.096 & $51.2(9.9)$ & $48.3(11.0)$ & $<0.001$ \\
\hline Rural & $42.5(11.8)$ & $42.3(12.8)$ & 0.804 & $49.0(11.5)$ & $48.3(11.3)$ & 0.296 \\
\hline
\end{tabular}

Notes: ${ }^{1} \mathrm{p}$-values are for comparison of difference of each category of patient and practice characteristics using independent $\mathrm{t}$ - test.

${ }^{2}$ Includes patients looking for work, studying full-time, looking after family and unable to work due to sickness or disability.

${ }^{3}$ Includes single/separated/divorced/widowed.

Number in each category is as shown in Table 1 subject to small number of missing values in SF12 items.

Unknowns: Age $=5$, Home and car ownership $=21$, Education $=11$, Employment $=10$, Marital status $=69$, Disease $=50$, Overall PACIC score $=52$

Patient characteristics were collected independently using PACIC for the same respondents.

Number of GPs is shown in Table 1.

The overall means of PCS-12 scores for male and female patients were $43.0(\mathrm{SD}=11.7)$ and $42.3(\mathrm{SD}=12.4)$ respectively (Table 2). Similarly, overall MCS-12 scores were $50.0(\mathrm{SD}=10.9)$ for men and $48.3(\mathrm{SD}=11.2)$ for women. Table 2 shows the differences between unadjusted PCS-12 or MCS-12 scores of male and female patients for the subcategories of patient and GP characteristics (Table 2). Women reported poorer physical health than men among those who had one chronic disease $(\mathrm{P}=0.035)$ and were under the care of a male GP $(P=0.014$, Table 2$)$. Unemployed females reported better physical health than unemployed males $(P=0.020)$. Similarly, women reported poorer mental health than men across the following patient characteristics: middle age $(\mathrm{P}=0.005)$, home and car owners $(P=0.007)$, employed $(P=0.003)$, two or more chronic diseases $(P=0.01)$, smokers $(P=0.001)$, high or low PACIC scores $(\mathrm{P}=0.03)$, under the care of a male GP $(\mathrm{P}=0.017)$, a GP who was Australian trained $(\mathrm{P}=0.001),<6(\mathrm{P}=0.028)$ or $>15$ years $(\mathrm{P}=0.003)$ as a GP in the current practice, $>24$ years as a GP $(\mathrm{P}=0.013)$, who worked in a larger practice $(\mathrm{P}=0.006)$ or in an urban area $(\mathrm{P}<0.001)$ (Table 2$)$. However, the above effects were not adjusted for confounding effects.

Gender differences in independent variables after adjustment for confounding effects with the multilevel regression analyses for each of the response variables are presented in Tables 3 and 4.

PCS-12 scores declined with age for both male $(\mathrm{P}<0.05)$ and female patients $(\mathrm{P}<0.01)$, but in contrast MCS-12 scores increased with age for female patients $(\mathrm{P}<0.001)$.
Both men and women with good general health were likely to have clinically significant better physical health with large effect sizes (> 1.3, B (regression coefficient) $>10.9, \mathrm{P}<0.001)$ and better mental health with a moderate effect size $(>0.7, \mathrm{~B}>6.8, \mathrm{P}<0.001)$ compared to those with poor general health (Tables 3 \& 4). Home and car ownership was positively associated with PCS-12 scores of female patients (effect size $=0.79, \mathrm{~B}=4.2, \mathrm{P}<0.01$ ) and MCS-12 scores of male patients (effect size $=0.82, \mathrm{~B}=3.4$, $\mathrm{P}<0.05$ ). Patients who were employed (effect size $=1.23$, $\mathrm{B}=7.3, \mathrm{P}<0.001$ ) or retired (effect size $=0.51, \mathrm{~B}=3.9$, $\mathrm{P}<0.001)$ were likely to have higher PCS-12 scores and MCS-12 scores (effect size $>0.55, \mathrm{~B}>3.4, \mathrm{P}<0.01$ ) than unemployed male patients. Employment was likely to have a positive effect on PCS-12 scores (effect size $=0.78, \mathrm{~B}=$ $4.1, \mathrm{P}<0.001)$ and retirement was likely to have a positive effect on MCS-12 scores (effect size $=0.52, \mathrm{~B}=2.9, \mathrm{P}<$ 0.01 ) for women. An effect size of more than 0.5 is considered to be clinically significant [3]. The number of chronic medical conditions was negatively associated with both MCS-12 $(\mathrm{P}<0.05)$ and PCS-12 scores $(\mathrm{P}<0.05)$ for women and with PCS-12 ( $<<0.001)$ for men. Female patients who were married or cohabiting tended to have higher MCS-12 scores than those who were not $(\mathrm{P}<0.001)$. Female smokers were likely to have a lower mental health score than non-smokers (effect size = $0.66, \mathrm{~B}=-4.0, \mathrm{P}<0.001)$. Neither smoking status nor marital status was associated with PCS-12 scores for any gender or MCS-12 scores for men. Results also showed a positive association between patient assessed 
Table 3 Regression coefficients (standard errors) of general practitioner and patient characteristics (number of general practitioners $=193$ ) for physical components score

\begin{tabular}{|c|c|c|c|c|}
\hline \multirow[t]{3}{*}{ Parameters (reference category) } & \multicolumn{4}{|c|}{ Multilevel estimates for the main model } \\
\hline & \multicolumn{2}{|c|}{ Male } & \multicolumn{2}{|c|}{ Female } \\
\hline & $\begin{array}{l}\text { Complete cases } \\
\quad(n=768)\end{array}$ & $\begin{array}{l}\text { Imputed full model } \\
\qquad(\mathrm{n}=1018)\end{array}$ & $\begin{array}{l}\text { Complete cases } \\
\qquad(\mathrm{n}=823)\end{array}$ & $\begin{array}{l}\text { Imputed full model } \\
\qquad(n=1163)\end{array}$ \\
\hline \multicolumn{5}{|l|}{ Fixed effects } \\
\hline Intercept & 35.89 & 34.61 & 37.60 & 36.47 \\
\hline \multicolumn{5}{|l|}{ Patient characteristics } \\
\hline \multicolumn{5}{|l|}{ Age, years } \\
\hline $40-59(18-39)$ & $-6.18(2.44)^{*}$ & $-4.43(2.39)$ & $-2.87(1.92)$ & $-1.40(1.60)$ \\
\hline$>59(18-39)$ & $-7.62(2.47)^{\dagger}$ & $-5.82(2.41)^{*}$ & $-5.75(1.96)^{\dagger}$ & $-4.52(1.62)^{\dagger}$ \\
\hline Good or very good health (very bad, bad or fair health) & $11.11(0.69)^{\ddagger}$ & $10.92(0.75)^{\ddagger}$ & $12.93(0.70)^{\ddagger}$ & $12.37(0.67)^{\ddagger}$ \\
\hline Own home \& car (rented \& don't own a car) & $2.14(1.72)$ & $2.79(1.52)$ & $4.07(1.45)^{\dagger}$ & $4.23(1.38)^{\dagger}$ \\
\hline Own home only (rented \& don't own car) & $-0.83(2.88)$ & $-0.38(2.98)$ & $1.56(1.69)$ & $2.03(1.65)$ \\
\hline Own car only (rented \& don't own a car) & $1.74(1.83)$ & $2.57(1.72)$ & $1.13(1.63)$ & $1.32(1.57)$ \\
\hline College / university (elementary / high school) & $1.68(0.73)^{*}$ & $1.65(0.70)^{*}$ & $-0.19(0.78)$ & $0.05(0.75)$ \\
\hline Employed patients (unemployed') & $7.66(1.15)^{\ddagger}$ & $7.29(1.04)^{\ddagger}$ & $4.22(0.99)^{\ddagger}$ & $4.05(1.08)^{\ddagger}$ \\
\hline Retired patients (unemployed') & $3.90(1.11)^{\ddagger}$ & $3.86(1.03)^{\ddagger}$ & $0.09(0.87)$ & $0.08(0.91)$ \\
\hline Married/cohabiting (single/separated/divorced/widowed) & $0.36(0.88)$ & $0.03(0.87)$ & $0.75(0.75)$ & $1.20(0.72)$ \\
\hline Two or more conditions (one condition) & $-2.20(0.68)^{\dagger}$ & $-2.57(0.69)^{\ddagger}$ & $-1.79(0.74)^{*}$ & $-1.56(0.72)^{*}$ \\
\hline Smoker (non-smoker) & $-0.54(1.25)$ & $-0.43(1.23)$ & $0.67(1.14)$ & $0.67(1.13)$ \\
\hline Overall satisfaction with care & $0.07(0.32)$ & $0.13(0.30)$ & $-0.84(0.33)^{*}$ & $-0.78(0.38)^{*}$ \\
\hline \multicolumn{5}{|l|}{ General Practitioner characteristics } \\
\hline Female (Male) & $1.52(0.98)$ & $1.48(1.04)$ & $2.19(0.80)^{\dagger}$ & $1.59(0.78)^{*}$ \\
\hline Overseas qualified (qualified in Australia) & $1.39(0.88)$ & $1.37(0.99)$ & $0.47(0.85)$ & $-0.09(0.83)$ \\
\hline Years in the current practice $6-15(<6)$ & $-0.27(0.91)$ & $-0.44(0.93)$ & $-0.80(0.89)$ & $-0.58(0.85)$ \\
\hline$>15(<6)$ & $0.02(0.90)$ & $0.08(0.92)$ & $1.05(0.95)$ & $1.20(1.05)$ \\
\hline \multicolumn{5}{|l|}{ Years as a GP } \\
\hline $19-24(<19)$ & $-0.80(0.95)$ & $-1.04(0.96)$ & $0.62(0.89)$ & $-0.43(0.87)$ \\
\hline$>24(<19)$ & $0.61(0.99)$ & $-0.04(1.0)$ & $-0.57(1.00)$ & $-0.91(1.01)$ \\
\hline Size 1-3 GPs in the practice (4 or more GPs) & $1.04(0.72)$ & $0.82(0.81)$ & $-1.54(0.75)^{*}$ & $-1.10(0.72)$ \\
\hline Urban practice (Rural practice) & $-0.18(0.70)$ & $-0.09(0.69)$ & $-0.27(0.76)$ & $-0.05(0.74)$ \\
\hline \multicolumn{5}{|l|}{ Random effects } \\
\hline Variance between general practitioners (standard errors) & $0.00(0.00)$ & $1.15(1.60)$ & $0.00(0.00)$ & $0.93(1.56)$ \\
\hline$\%$ variance explained & 100 & 70.2 & 100 & 90.3 \\
\hline Variance between patients (standard errors) & $81.84(4.18)^{\ddagger}$ & $84.66(4.81)^{\ddagger}$ & $91.49(4.51)^{\ddagger}$ & $93.29(5.21)^{\ddagger}$ \\
\hline$\%$ variance explained & 38.6 & 36.3 & 37.4 & 36.3 \\
\hline
\end{tabular}

Note:* $\mathrm{P}<0.05,^{\dagger} \mathrm{P}<0.01,^{\ddagger} \mathrm{P}<0.001$

${ }^{1}$ Includes patients looking for work, studying full-time, looking after family and unable to work due to sickness or disability.

Patient characteristics were collected independently using PACIC for the same respondents.

quality of care and MCS-12 scores $(\mathrm{P}<0.05)$ and a negative association between quality of care and PCS12 scores $(\mathrm{P}<0.05)$ for females only. Effect sizes for some of the significant effects (for example, number of conditions, satisfaction with care, married status) were small $(<0.5)$ and clinically not significant. Being under the care of a female GP was likely to have a positive effect on PCS-12 scores $(\mathrm{P}<0.05)$ of female patients. Other GP characteristics were not associated with either PCS-12 or MCS-12 scores for either gender.

\section{Variance explained}

At the GP level (level 2), $70 \%$ and $90 \%$ of the variances in PCS-12 scores between GPs for male and female 
Table 4 Regression coefficients (standard errors) of general practitioner and patient characteristics (number of general practitioners $=193$ ) for mental health components score

\begin{tabular}{|c|c|c|c|c|}
\hline \multirow[t]{3}{*}{ Parameters (reference category) } & \multicolumn{4}{|c|}{ Multilevel estimates for the main model } \\
\hline & \multicolumn{2}{|c|}{ Male } & \multicolumn{2}{|c|}{ Female } \\
\hline & $\begin{array}{l}\text { Complete cases } \\
\quad(n=768)\end{array}$ & $\begin{array}{l}\text { Imputed full model } \\
\qquad(n=1018)\end{array}$ & $\begin{array}{l}\text { Complete cases } \\
\qquad(\mathrm{n}=823)\end{array}$ & $\begin{array}{l}\text { Imputed full model } \\
\qquad(n=1163)\end{array}$ \\
\hline \multicolumn{5}{|l|}{ Fixed effects } \\
\hline Intercept & 35.52 & 35.09 & 32.80 & 34.16 \\
\hline \multicolumn{5}{|l|}{ Patient characteristics } \\
\hline \multicolumn{5}{|l|}{ Age, years } \\
\hline $40-59(18-39)$ & $0.48(2.64)$ & $0.31(2.49)$ & $2.83(1.96)$ & $2.42(1.57)$ \\
\hline$>59(18-39)$ & $1.89(2.68)$ & $1.68(2.51)$ & $6.33(1.99)^{\dagger}$ & $5.62(1.64)^{\ddagger}$ \\
\hline Good or very good health (very bad, bad or fair health) & $6.92(0.75)^{\ddagger}$ & $6.84(0.79)^{\ddagger}$ & $7.31(0.71)^{\ddagger}$ & $7.33(0.62)^{\ddagger}$ \\
\hline Own home \& car (rented \& don't own car) & $5.04(1.87)^{\dagger}$ & $3.42(1.71)^{*}$ & $1.37(1.48)$ & $1.50(1.29)$ \\
\hline Own home only (rented \& don't own a car) & $3.24(3.12)$ & $0.30(2.77)$ & $0.47(1.72)$ & $0.27(1.61)$ \\
\hline Own car only (rented \& don't own car) & $3.04(1.98)$ & $1.55(1.93)$ & $0.67(1.66)$ & $0.67(1.43)$ \\
\hline College / university (elementary / high school) & $0.78(0.79)$ & $0.65(0.78)$ & $0.07(0.79)$ & $0.00(0.72)$ \\
\hline Employed patients (unemployed') & $2.95(1.24)^{*}$ & $3.40(1.18)^{\dagger}$ & $1.42(1.01)$ & $1.16(1.03)$ \\
\hline Retired patients (unemployed') & $4.95(1.20)^{\ddagger}$ & $5.43(1.10)^{\ddagger}$ & $2.62(0.89)^{+}$ & $2.86(0.87)^{\dagger}$ \\
\hline Married/cohabiting (single/separated/divorced/widowed) & $0.91(0.95)$ & $0.96(1.03)$ & $2.29(0.77)^{\dagger}$ & $2.43(0.73)^{\ddagger}$ \\
\hline Two or more conditions (one condition) & $0.11(0.74)$ & $-0.05(0.77)$ & $-0.96(0.76)$ & $-1.56(0.73)^{*}$ \\
\hline Smoker (non-smoker) & $1.62(1.35)$ & $1.16(1.39)$ & $-4.20(1.17)^{\ddagger}$ & $-3.99(1.08)^{\ddagger}$ \\
\hline Overall satisfaction with care & $0.60(0.35)$ & $0.56(0.36)$ & $1.04(0.34)^{\dagger}$ & $0.84(0.34)^{*}$ \\
\hline \multicolumn{5}{|l|}{ General Practitioner characteristics } \\
\hline Female (Male) & $0.55(1.06)$ & $0.41(1.04)$ & $-1.25(0.81)$ & $-0.74(0.75)$ \\
\hline Overseas qualified (qualified in Australia) & $-1.87(0.96)$ & $-1.73(0.98)$ & $-0.28(0.86)$ & $0.02(0.85)$ \\
\hline \multicolumn{5}{|l|}{ Years in the current practice } \\
\hline $6-15(<6)$ & $-1.32(0.99)$ & $-0.82(0.97)$ & $0.66(0.91)$ & $0.90(0.88)$ \\
\hline$>15(<6)$ & $-0.04(0.97)$ & $0.02(0.99)$ & $0.18(0.97)$ & $0.36(1.02)$ \\
\hline \multicolumn{5}{|l|}{ Years as a GP } \\
\hline $19-24(<19)$ & $0.35(1.03)$ & $0.51(1.04)$ & $0.88(0.91)$ & $0.49(0.87)$ \\
\hline$>24(<19)$ & $0.58(1.07)$ & $0.44(1.13)$ & $0.23(1.02)$ & $-0.52(1.04)$ \\
\hline Size $1-3$ GPs in the practice (4 or more GPs) & $-0.84(0.78)$ & $-0.79(0.80)$ & $-0.63(0.77)$ & $-0.69(0.69)$ \\
\hline Urban practice (Rural practice) & $0.87(0.76)$ & $0.93(0.74)$ & $-0.34(0.78)$ & $-0.32(0.73)$ \\
\hline \multicolumn{5}{|l|}{ Random effects } \\
\hline Variance between general practitioners (standard errors) & $0.00(0.00)$ & $0.92(1.53)$ & $0.00(0.00)$ & $0.30(1.01)$ \\
\hline$\%$ variance explained & 100 & 84.3 & 100 & 88.1 \\
\hline Variance between patients (standard errors) & $96.08(4.90)^{\ddagger}$ & $97.67(5.35)^{\ddagger}$ & $95.10(4.69)^{\ddagger}$ & $96.36(4.43)^{\ddagger}$ \\
\hline$\%$ variance explained & 16.6 & 16.4 & 22.9 & 22.3 \\
\hline
\end{tabular}

Note: ${ }^{*} \mathrm{P}<0.05,{ }^{\dagger} \mathrm{P}<0.01,{ }^{\ddagger} \mathrm{P}<0.001$.

${ }^{1}$ Includes patients looking for work, studying full-time, looking after family and unable to work due to sickness or disability.

Patient characteristics were collected independently using PACIC for the same respondents.

patients were explained respectively by the variables used in the analysis (Table 3). At the patient level (level 1) the variance in PCS-12 scores explained was similar (36\%) for male and female patients (Table 3). Similarly, for MCS-12 scores, GP level variances explained were $84 \%$ for males and $88 \%$ for females and patient level variances explained were $16 \%$ for males and $22 \%$ for female patients (Table 4).

\section{Discussion}

This study is one of very few to explore gender differences in HRQoL among patients with type 2 diabetes 
and hypertension/ischaemic heart disease. The SF-12 is a subjective measure of health that can be influenced by a respondent's perceptions, expectations and interpretations about health [6]. Nonetheless, the scale has become one of the most widely used HRQoL measures. This study provides comprehensive data on how patient and GP characteristics predict self-rated physical and mental health of chronically-ill patients in Australia.

The results show that chronically-ill women smokers reported clinically significantly (effect size $=0.66$ ) poorer mental health than non-smokers. This supports findings from previous studies showing that the association between smoking and HRQoL is different between women and men [13,30,31]. Lasser et al. [10] suggested that people with poor mental health are more likely to smoke than those who have good mental health. A higher physical and psychological dependence among women is a possible explanation for the increased mental distress observed among women who unsuccessfully attempted to quit [32]. Strine et al. [8] found a significant association between smoking and impaired mental health. The provision of psychological care in conjunction with smoking-cessation programs, and vice versa, is indicated [33]. Smoking is one of the strongest modifiable risk factors for a host of health outcomes that contribute to morbidity and mortality in Australia and worldwide in chronically-ill patients. All this suggests that chronicallyill women may need more psychological support in their attempts to quit smoking. Smoking is one of the most significant risk factors for the development of cardiovascular disease in diabetes patients [34]. Smoking is a risk factor for mortality and coronary heart disease in hypertension and in diabetes [35]. Chronically ill, particularly females with mental illness are motivated to attend smoking reduction and cessation programmes. When supported programmes providing nicotine replacement and counseling are offered to people with mental illness, it has been found that they are able to quit smoking at equivalent rates to the general population [36]. It is important to ensure that the person with severe mental illness is aware of the risks of smoking because basic medical education is frequently missing in this patient group [37]. Integrated care is desirable, as psychiatric symptoms may be exacerbated and severe withdrawal symptoms experienced by smokers with a mental illness undertaking smoking cessation treatment. When smoking cessation and psychiatric care is integrated these adverse effects appear to be reduced [38]. The ability to detect hypothesized relationship in previous studies between smoking and mental health of females suggests construct validity of smoking status in this study.

The results show that women under the care of female GPs reported better physical health (effect size $=0.30$ ) than those under the care of male GPs. Physician's gender is one of many factors that impact on the doctor patient interaction [16]. As noted in previous studies, female GPs tend to have longer consultations [15,39], especially with their female patients. Female patients often report that they prefer female doctors for female-specific health problems $[40,41]$, intimate problems $[42,43]$, behavioral problems [41], and endocrinologic/metabolic problems [14]. Female physicians were found to be more likely to perform female-specific prevention procedures, check patients' blood pressure and make some follow-up arrangements and referrals [44]. Also, compared to their male colleagues, female physicians were shown to use a more participatory decision-making process [45] that encourages self-management, which is a fundamental part of diabetes care and may result in improved diabetes control [46].

Socio-demographic differences between male and female patients in the current study (e.g., the women were younger, less likely to be married, and had lower socioeconomic status than the men) are consistent with those found in other similar studies $[47,48]$ and provide an understanding of gender differences in HRQoL.

Our study showed that there are gender differences in how patient characteristics impact on self-assessed physical and mental health. For example, previous research found that lower socio-economic groups reported lower PCS-12 and MCS-12 [4,5,49]. Our results showed that home and car ownership tended to have a positive effect on the self-assessed physical health of women but not men and on the mental health of men, but not women. Some studies have shown a significant interaction effect between gender and employment, indicating that employed men enjoyed higher levels of general wellbeing $[2,7,50]$. In this study the negative impact of unemployment was likely to be greater in male than female patients. Unemployment was likely to have a larger negative effect on HRQoL of men than that of women. This may be because the significance of work and its impact on household income may be greater in chronically-ill older men than in women [51]. Also the observed age difference in HRQoL differed among men and women. The younger men $(<39$ years) were likely to report better physical health than the older men (> 59 years), whereas the older women reported better mental health than the younger women.This result is consistent with previous studies. For example, Hanmer et al. [52] reported similar mental health (MCS-12) mean scores for younger men (< 39 years, 52.1 ) and older men (> 59 years, 52.3) whereas older women reported better mental health mean scores (51.3) than younger women (49.6).

Two hundred and eleven patients suffered from only type 2 diabetes and 793 from type 2 diabetes and hypertension and/or ischemic heart disease. Further 1129 
suffered from hypertension and/or ischemic heart disease. The overall mean PCS-12 of male diabetes only patients $(46.7, \mathrm{SD}=12.4)$ and male hypertension/ischaemic heart disease only patients $(44.8, \mathrm{SD}=11.5)$ in the study were less than male U.S. general population (mean = 50.6, SD = 10.2) [19]. The difference for PCS-12 was clinically not significant for diabetes (effect size $=0.38<$ 0.5 ), whereas it was clinically significant (effect size $=$ 0.56) for hypertension/ischaemic heart disease. Similarly, the overall mean PCS-12 of female diabetes only patients $(44.7, \mathrm{SD}=11.6)$ and female hypertension/ischaemic heart disease patients $(43.4, \mathrm{SD}=12.6)$ were less than female U.S. general population (mean $=48.7$, $\mathrm{SD}=9.6)$ [19]. The difference was clinically not significant for diabetes (effect size $=0.41$ ), whereas for hypertension/ischaemic heart disease patients, the difference was clinically significant (effect size $=0.53$ ). However, mean MCS-12 of male (49.3, SD = 10.6) and female (47.7, SD = 12.3 ) diabetes only patients and male $(50.5, \mathrm{SD}=10.5)$ and female (49.2, $\mathrm{SD}=10.8)$ hypertension/ischaemic heart disease patients was not clinically significant compared to male (50.4, SD = 9.9) and female (48.4, SD = 9.6) MCS-12 scores of U.S. general population.

Although the GP level variance explained was very high (from $70 \%$ to $90 \%$ ) for both summary scores, the patient level variance explained for mental health was half that of physical health (36\%). There may have been other lifestyle and clinical risk factors important to mental health assessment which were not specifically evaluated in this study and warrant further exploration in the Australian context.

There are a number of limitations to this study. Patients that the practice identified as being unable to read English were excluded from the study. Although the response rate of $70 \%$ was comparable with other studies [53], it is possible that non-responders might have assessed their physical and mental health differently from those who responded. We were unable to analyse differences between respondents and non-respondents in the study as information about non-respondents could not be captured as recruitment was at arm's length through practices. In a similar HRQoL study in Australia with 7606 chronically-ill patients from 96 general practices, the response rate was $61 \%$ [2]. In that study we had gender for non-respondents. We conducted analyses comparing proportions of respondents with nonrespondents for gender. The gender of respondents (53.3\% were females) and non-respondents $(53.6 \%$ were females) were similar $(\mathrm{P}=0.76)$. As females attend a GP more often than males, they have a greater chance of being selected in the sample [54]. Male (46.7\%) and female (53.3\%) patients responded to mental health questions in the study were similar to other studies [2]. Further, the socio-economic status of male patients were similar in both studies (home owners: $82.3 \%$ vs. $81.4 \%$ ). We compared patient characteristics of this study with a similar Australian general practice study with type 2 diabetes, ischaemic heart disease/hypertension and asthma patients [55]. The proportions of gender $(\mathrm{P}=0.712)$, home ownership $(\mathrm{P}=0.690)$, and marital status $(\mathrm{P}=$ 0.903 ) were similar between the two studies (data not shown). However, the patients in this study were older $(69 \%$ vs. $55 \%$ for $>59$ years, $\mathrm{P}<0.01)$ and marginally less employed ( $32 \%$ vs. $34 \%, \mathrm{P}=0.024)$. The reason being that, in the other study asthma patients $(\mathrm{n}=724)$ were much younger (mean $=50$ years) and our study does not have any asthma patients. The mean age in our study was 64 years. Hence our study had more older and retired ( $47 \%$ vs. $40 \%$ ), and therefore less employed patients.

In Australia, the proportion of males and females who smoked declined $1.4 \%$ (22.5\% in 2004 and $21.1 \%$ in 2007 ) and $1.1 \%$ (18.8\% in 2004 and $17.7 \%$ in 2007$)$ respectively between 2004 and 2007 [56]. In Australia, the proportion of males and females type 2 diabetes and IHD patients who smoked in 2007 were $13.9 \%$ and $15.3 \%$ respectively [57]. Those figures were slightly higher than the proportions for type 2 diabetes and/or IHD patients for males $(9.2 \%)$ and females $(11.1 \%)$ of our study.

There were no significant differences in GP characteristics between our sample and all GPs in Australia in terms of female gender (37.3\% vs. $35.9 \%)$. This proportion was similar in Beech study (36.8\%) [54]. However, GPs who graduated in a country other than Australia were slightly under-represented when compared with Australian total sample $(25.5 \%$ vs. $30.2 \%)$ but not with those participated in Beech study (26.5\%) [54]. In Australia, patients can choose what doctor they see, at whichever practice they choose. There are no patient boundaries and patients do not have to 'sign up' with a particular practice.

The actual ICC computed for MCS-12 from the final multilevel model is 0.003 for females and 0.009 for males which is lower than that used in the power calculations. With actual ICC, 960 patients from each gender were adequate to detect an effect size of 0.13 between smokers and non-smokers.

Strengths of the study include the large number of patients and GPs participating, the adjustment for confounding patient and GP factors, the correction for GP-level clustering with multilevel modeling, and addressing the potential bias and loss of precision arising from missing values using multiple imputations.

\section{Conclusions}

The increased prevalence of poorer self-assessed mental health status among chronically ill women who smoke is deserving of further investigation. Better understanding of the associations between HRQoL and smoking may help to reduce barriers to smoking cessation and help to 
better tailor smoking cessation support to patients' needs, ultimately improving their overall well-being. This is especially important in the context of chronic disease, where improvement of lifestyle behavior is central to management. Clinicians are advised to pay more attention to the mental health status of chronically-ill smokers, specially women.

More quantitative studies are needed to explore the interaction between GP gender and patient gender in relation to HRQoL.

\section{Abbreviations}

B: Regression coefficient; CCl: Clinical care interview; BP: Bodily pain; GH: General health; GP: General practitioner; HRQOL: Health-related quality of life; ICC: Intra-cluster correlation; MCS: Mental Health Component Summary; MCS-12: Mental Component Score derived from the SF-12; MH: Mental health; PACIC: Patient Assessment of Chronic Illness Care; PCS: Physical Component Summary; PCS-12: Physical Component Score derived from the SF-12; PF: Physical functioning; RE: Role emotional; RP: Role physical; SD: Standard deviation; SF: Social Functioning; SF-12: Short Form 12-item Health Survey; SF-36: Short Form 36-item Health Survey; VT: Vitality.

\section{Competing interests}

The authors declare that they have no competing interests.

\section{Authors' contributions}

UJ contributed to data analysis, interpreting the data and drafting the manuscript. UJ and $\mathrm{MH}$ made substantial contributions to the conception and design of the study. JT and BC were involved in the data collection. All authors were involved in drafting the manuscript or revising it critically for important intellectual content. All authors have read and approved the final version of the manuscript.

\section{Acknowledgements}

The authors would like to thank all patients, practices and their staff who participated in this study and participating Divisions of General Practice. We would like to acknowledge Judy Proudfoot, Jocelyn Tan, Gawaine Powell Davies, David Perkins, and Mahnaz Fanaian. We are grateful to Ms Joan Rosenthal for her advice and detailed editorial assistance.

\section{Author details}

${ }^{1}$ Centre for Primary Health Care and Equity, University of New South Wales, Sydney, New South Wales, Australia. ${ }^{2}$ Westmead Breast Cancer Institute, Westmead, New South Wales, Australia. ${ }^{3}$ Faculty of Health Sciences, University of Sydney, Lidcombe, New South Wales, Australia.

Received: 7 January 2013 Accepted: 17 June 2013

Published: 21 June 2013

\section{References}

1. Australian Institute of Health and Welfare: Chronic disease and associated risk factors in Australia. Canberra: AlHW; 2006.

2. Jayasinghe UW, Proudfoot J, Barton CA, Amoroso C, Holton C, Davies GP, Beilby J, Harris MF: Quality of life of Australian chronically-ill adults: patient and practice characteristics matter. Health Qual Life Outcomes 2009, 7:50.

3. Lim LLY, Fisher JD: Use of the 12-item Short-Form (SF-12) Health Survey in an Australian heart and stroke population. Qual Life Res 1999, $8(1-2): 1-8$.

4. Fone D, Dunstan F, Lloyd K, Williams G, Watkins J, Palmer S: Does social cohesion modify the association between area income deprivation and mental health? A multilevel analysis. Int J Epidemiol 2007, 36(2):338-345.

5. Burdine JN, Felix MRJ, Abel AL, Wiltraut CJ, Musselman YJ: The SF-12 as a population health measure: an exploratory examination of potential for application. Health Serv Res 2000, 35(4):885-904.

6. Keles H, Ekici A, Ekici M, Bulcun E, Altinkaya V: Effect of chronic diseases and associated psychological distress on health-related quality of life. Internal Med J 2007, 37(1):6-11.
7. Lennon MC: Sex differences in distress: the impact of gender and work roles. J Health Soc Behav 1987, 28(3):290-305.

8. Strine TW, Okoro CA, Chapman DP, Balluz LS, Ford ES, Ajani UA, Mokdad AH: Health-related quality of life and health risk behaviors among smokers. Am J Prev Med 2005, 28(2):182-187.

9. Jiang Y, Hesser JE: Associations between health-related quality of life and demographics and health risks: results from Rhode Island's 2002 behavioral risk factor survey. Health Qual Life Outcomes 2006, 4:14.

10. Lasser K, Boyd JW, Woolhandler S, Himmelstein DU, McCormick D, Bor DH: Smoking and mental illness: a population-based prevalence study. JAMA 2000, 284(20):2606-2610.

11. Hanmer J, Hays RD, Fryback DG: Mode of administration is important in US national estimates of health-related quality of life. Med Care 2007, 45(12):1171-1179.

12. Jia H, Lubetkin El, Moriarty DG, Zack MM: A comparison of healthy days and EuroQol EQ-5D measures in two US adult samples. Appl Res Qual Life 2007, 2(3):209-221.

13. Wilson DH, Chittleborough CR, Kirke K, Grant JF, Ruffin RE: The healthrelated quality of life of male and female heavy smokers. Sozial- und Praventivmedizin 2004, 49(6):406-412.

14. Bensing JM, van den Brink-Muinen A, de Bakker DH: Gender differences in practice style: a Dutch study of general practitioners. Med Care 1993, 31(3):219-229

15. Roter D, Lipkin M Jr, Korsgaard A: Sex differences in patients' and physicians' communication during primary care medical visits. Med Care 1991, 29(11):1083-1093.

16. Bertakis KD: The influence of gender on the doctor-patient interaction. Patient Ed Couns 2009, 76(3):356-360

17. Taggart J, Chan B, Jayasinghe UW, Christl B, Proudfoot J, Crookes P, Beilby J, Black D, Harris MF: Patients Assessment of Chronic Illness Care (PACIC) in two Australian studies: structure and utility. J Eval Clin Pract 2011, 17(2):215-221

18. Jenkinson C, Chandola T, Coulter A, Bruster S: An assessment of the construct validity of the SF-12 summary scores across ethnic groups. J Pub Health Med 2001, 23(3):187-194.

19. Ware JEJ, Kosinski M, Turner-Bowker DM, Gandek B: How to score Version 2 of the SF-12 Health Survey (with a supplement Documenting Version 1). Lincoln, Rl: QualityMetric Incorporated; 2002

20. Schofield MJ, Mishra G: Validity of the SF-12 compared with the SF-36 Health Survey in pilot studies of the Australian Longitudinal Study on Women's Health. J Health Psychol 1998, 3(2):259-271.

21. Gandek B, Ware JE, Aaronson NK, Apolone G, Bjorner JB, Brazier JE, Bullinger M, Kaasa S, Leplege A, Prieto L, Sullivan M: Cross-validation of item selection and scoring for the SF-12 Health Survey in nine countries: results from the IQOLA project. J Clin Epidem 1998, 51(11):1171-1178.

22. Sanderson $\mathrm{K}$, Andrews G: The SF-12 in the Australian population: crossvalidation of item selection. Aust NZ J Pub Health 2002, 26(4):343-345.

23. Wilson D, Tucker G, Chittleborough C: Rethinking and rescoring the SF-12. Sozial- und Praventivmedizin 2002, 47(3):172-177.

24. Macintyre S, Ellaway A, Der G, Ford G, Hunt K: Do housing tenure and car access predict health because they are simply markers of income or self esteem? A Scottish study. J Epidem Commun Health 1998, 52(10):657-664

25. Kontodimopoulos N, Pappa E, Niakas D, Tountas Y: Validity of SF-12 summary scores in a Greek general population. Health Qual Life Outcomes 2007, 5:55

26. Cohen J: Statistical power analysis for the behavioral sciences. New York: Academic Press: 1977.

27. Rasbash J, Browne W, Healy M, Cameron B, Charlton C: A user's guide to MLwiN version 2.25. University of Bristol: Centre for Multilevel Modelling; 2012.

28. Carpenter JR, Goldstein H, Kenward MG: REALCOM-IMPUTE software fo multilevel multiple imputation with mixed response types. J Stat Software 2011, 45(5):1-14

29. Robin DB: Multiple imputation for non-response in surveys. New York: Wiley; 1987.

30. Bjornson W, Rand C, Connett JE, Lindgren P, Nides M, Pope F, Buist AS, Hoppe-Ryan C, O'Hara P: Gender differences in smoking cessation after 3 years in the Lung Health Study. Am J Pub Health 1995, 85(2):223-230.

31. Reynoso J, Susabda A, Cepeda-Benito A: Gender differences in smoking cessation. J Psychopathol Behav 2005, 27(3):227-234. 
32. McClave AK, Dube SR, Strine TW, Mokdad AH: Associations between health-related quality of life and smoking status among a large sample of U.S. adults. Prev Med 2009, 48(2):173-179.

33. Jiang $Y$, Hesser JE: Patterns of health-related quality of life and patterns associated with health risks among Rhode Island adults. Health Qual Life Outcomes 2008, 6:49.

34. Turner RC: The U.K. prospective diabetes study: A review. Diabetes Care 1998, 21(3):C35-c38.

35. Fagard $\mathrm{RH}$ : Smoking amplifies cardiovascular risk in patients with hypertension and diabetes. Diabetes Care 2009, 32(Suppl. 2):S429-S431.

36. Coghlan R, Lawrence D, Holman CDJ, Jablensky AV: Duty to Care: Physical illness in people with mental illness. Perth: The University of Western Australia; 2001

37. Holt RIG, Peveler RC: Diabetes and cardiovascular risk in severe mental illness: a missed opportunity and challenge for the future. Practical Diabetes Int 2010, 27(2):79-84.

38. McFall M, Saxon AJ, Malte CA, Chow B, Bailey S, Baker DG, Beckham JC Boardman KD, Carmody TP, Joseph AM, Smith MW, Shih MC, Lu Y, Holodniy M, Lavori PW: Integrating tobacco cessation into Mental Health Care for post traumatic stress disorder: A randomised controlled trial. JAMA 2010, 304(22):2485-2493.

39. Hall JA, Irish JT, Roter DL, Ehrlich CM, Miller LH: Gender in medical encounters: an analysis of physician and patient communication in a primary care setting. Health Psychol 1994, 13(5):384-392.

40. Haar E, Halitsky V, Stricker G: Factors related to the preference for a female gynecologist. Med Care 1975, 13(9):782-790.

41. Fennema K, Meyer DL, Owen N: Sex of physician: patients' preferences and stereotypes. J Fam Pract 1990, 30(4):441-446.

42. Ackerman-Ross FS, Sochat N: Close encounters of the medical kind: attitudes toward male and female physicians. Soc Sci Med 1980, 14(1):61-64

43. Zare N, Sorenson JR, Heeren T: Sex of provider as a variable in effective genetic counseling. Soc Sci Med 1984, 19(7):671-675.

44. Franks $P$, Bertakis KD: Physician gender, patient gender, and primary care. J Women's Health 2003, 12(1):73-80.

45. Cooper-Patrick L, Gallo JJ, Gonzales JJ, Vu HT, Powe NR, Nelson C, Ford DE: Race, gender, and partnership in the patient-physician relationship. JAMA 1999, 282(6):583-589.

46. McKinlay JB, Lin T, Freund K, Moskowitz M: The unexpected influence of physician attributes on clinical decisions: results of an experiment. $J$ Health Soc Behav 2002, 43(1):92-106.

47. Skinner K, Sullivan LM, Tripp TJ, Kressin NR, Miller DR, Kazis L, Casey V: Comparing the health status of male and female veterans who use VA health care: results from the VA Women's Health Project. Women Health 1999, 29(4):17-33.

48. Robert SA, Cherepanov D, Palta M, Dunham NC, Feeny D, Fryback DG: Socioeconomic status and age variations in health-related quality of life: results from the national health measurement study. J Gerontol B Psychol Sci Soc Sci 2009, 64(3):378-389.

49. Wainwright NWJ, Surtees PG: Places, people, and their physical and mental functional health. J Epidemiol Commun Health 2004, 58(4):333-339.

50. Artazcoz L, Benach J, Borrell C, Cortès I: Unemployment and mental health: understanding the interactions among gender, family roles, and social class. Am J Pub Health 2004, 94(1):82-88.

51. Stolzenberg RM: It's about time and gender: spousal employment and health. Am J Sociol 2001, 107(1):61-100.

52. Hanmer J, Lawrence WF, Anderson JP, Kaplan RM, Fryback DG: Report of nationally representative values for the noninstitutionalized US adult population for 7 health-related quality-of-life scores. Med Decision Making 2006, 26(4):391-400.

53. Fone DL, Lloyd K, Dunstan FD: Measuring the neighbourhood using UK benefits data: a multilevel analysis of mental health status. BMC Pub Health 2007, 7:69.

54. Britt H, Miller GC, Charles J, Henderson J, Bayram C, Harrison C, Valenti L, Fahridin S, Pan Y, O'Halloran J: General practice activity in Australia 2007-08. General practice series no. 22. Cat. no. GEP 22. Canberra: Australian Institute of Health and Welfare; 2008.
55. Jayasinghe UW, Proudfoot J, Holton C, Powell Davies G, Amoroso C, Bubner T, Beilby J, Harris MF: Chronically ill Australians' satisfaction with accessibility and patient-centredness. Int J Qual Health Care 2008, 20:105-114.

56. Australian Institute of health and welfare: National drug strategy household survey. Canberra: AlHW: 2007

57. Australian Institute of Health and Welfare: Australia's health series no.13. Cat no. AUS 156. Canberra: AlHW; 2012

doi:10.1186/1477-7525-11-102

Cite this article as: Jayasinghe et al:: Gender differences in health-related quality of life of Australian chronically-ill adults: patient and physician characteristics do matter. Health and Quality of Life Outcomes 2013 11:102.

\section{Submit your next manuscript to BioMed Central and take full advantage of:}

- Convenient online submission

- Thorough peer review

- No space constraints or color figure charges

- Immediate publication on acceptance

- Inclusion in PubMed, CAS, Scopus and Google Scholar

- Research which is freely available for redistribution

Submit your manuscript at www.biomedcentral.com/submit
C Biomed Central 